\title{
Viejo y nuevo caciquismo durante los años treinta en Cádiz
}

\section{JOSE LUIS RODRIGUEZ MOLINA}

El caciquismo antes de la República, 1; La permanencia del caciquismo, 5; Una sòciedad a tres bandas, 8: -el caciquismo tradicional, 8; las clases medias emergentes, 12; la clase obrera, 14-; La ruptura del caciquismo, 16.

Aunque, generalmente, cuando se habla de caciquismo se hace referencia, de forma exclusiva, a un fenómeno de corrupción electoral, en realidad, este sistema es un modo de vida completo, en el que junto al empleo de procedimientos fraudulentos para lograr la victoria en unas elecciones, existe un conjunto de relaciones, entre el cacique y su cliente, que comprende otros ámbitos de sus actividades. En este sentido, José Varela Ortega afirma que el sistema caciquil prefiere el acuerdo antes que la lucha, en la que tiene que emplear el pucherazo, la compra de votos o la coacción que representan signos de su deterioro. Por el contrario, el cacique prefiere asentar su poder en la ignorancia, la dependencia económica y el control social de sus clientes. Situación que le dotan de una permanencia más prolongada, constituyéndose, incluso', como un hecho natural que llega a ser asumido, como tal, por la sociedad ${ }^{1}$.

Se afirma que,' con la proclamación de la Segunda República, el sistema caciquil entró en completa descomposición, aunque, durante esos años, persistieran fenómenos de clientelismo y corrupción propios de épocas anteriores. Sobre todo, en zonas en las que la desmovilización social, incluyendo la electoral, y el predominio de una oligarquía, financiera, mercantil y política, había sido una constante. Este es el caso de Cádiz, tanto la capital, sujeto de la presente comunicación, como la provincia.

\section{EL CACIQUISMO ANTES DE IA REPUBLICA}

Durante el reinado de Alfonso XIII, desde 1902, hasta la instauración de la dictadura de Primo de Rivera, en 1923, los distritos gaditanos

(1) José Varela Ortega, -Los amigos políticos: Funcionamiento del sistema caciquista•, Revista de Occidente, n.127, octubre, Madrid, 1973, págs.45-73. 
registraron tanto la presencia de una fuerte oligarquía terrateniente y mercantil, como un comportamiento electoral desmovilizador. Javier Tusell Gómez ha señalado que seis de ellos se encuentran entre los que, o bien no existió una efectiva lucha electoral, o el resultado arrojó la victoria del candidato gubernamental ${ }^{2}$.

Tampoco se notó en Cádiz la pretendida labor regeneracionista que el Dictador quiso imponer con su golpe de Estado. Bien relacionado con los viejos caciques, la disolución de las diputaciones, sólo sirvió para que otros nuevos, más o menos enlazados con los anteriores, los sustituyeran. Pero además, se puede decir que la Dictadura de Primo de Rivera no solo no eliminó a los profesionales sin escruípulos de la política, sino que, por el contrario, o bien pactó con ellos o mantuvo buenas relaciones, como las que permitieron la alcaldía de Ramón de Carranza en la capital gaditana.

Con estos antecedentes no puede extrañar que Cádiz fuera la única capital de provincia andaluza en la que venciera la candidatura monárquica en las elecciones de abril de $1931^{3}$. Además de la existencia de fraude -manipulación del censo, compra de votos y coacciones- como aseguraron los contemporáneos y ha recogido la historiografía actual ${ }^{4}$, se puede afirmar que se daban las condiciones para que el caciquismo tradicional, presente en la ciudad, hubiera vencido sin necesidad de recurrir a métodos extra-legales.

(2) El número de elecciones en las que no existió una auténtica lucha electoral por no presentarse más que un único candidato o porque, cuando hubo más de uno, éste no obtuvo más del $5 \%$ del total de electores fue de 11 en Medina Sidonia, 9 en Grazalema y Sanlúcar de Barrameda, 8 en Algeciras, 5 en El Puerto de Santa María y 2 en Jerez de la Frontera.

El diputado electo pertenecía al mismo partido político que el presidente del Consejo de ministros que convocó las elecciones fue en 15 ocasiones en El Puerto de Santa María, 14 en Medina Sidonia, 13 en Sanlúcar de Barrameda y 11 veces en Algeciras y 10 en Jerez de la Frontera.

En Javier Tusell Gómez, •El sistema caciquil andaluz comparado con otras regiones españolas (1903-1923)., Revista española de Investigaciones Sociológicas, n.2, abril-junio, Madrid, 1978, págs.12 y 14 .

(3) Para las elecciones de abril de 1931 se pueden consultar, Diego Caro Cancela, La Segunda República en Cádiz. Elecciones y partidos políticos, Cádiz, 1987, págs.57-84 y Shlomo Ben-Ami, Los origenes de la Segunda república española: Anatomía de una transición, Madrid, 1990, págs.434-456.

(4) Javier Tusell Gómez recoge las denuncias aparecidas en el periódico republicano - Libertad. de Cádiz: El soborno, la coacción, la compra de votos, la desvergüenza mayor conocida en las artes electorales fueron puestas en juego para derrotar la candidatura de la Coalición republicano-socialistan. Aparecieron el 20 de junio de 1931. En Javier Tusell Gómez, La crisis del caciquismo andaluz (1923-1931), Madrid, 1977. También en Diego Caro Cancela, La Segunda Repüblica en Cádiz. Elecciones y partidos políticos, Cádiz, 1987, pág.77. 
Parece cierto que el fenómeno caciquil se da con mayor intensidad en el mundo rural, mientras que decrece en el urbano. Es así por la existencia en el segundo de una mayor movilización social que incluye un variado cruce de intereses económicos y presencia de fuerzas al margen del sistema político, que supone tanto la existencia de una opinión priblica como la protesta, por ejemplo, contra la corrupción administrativa.

En Cádiz, a mediados de 1930, existía un débil republicanismo que no fue capaz de aprovechar el escándalo que supuso el denominado “Expediente Barahona*; algunos grupos masónicos, más dedicados a sus actividades internas que a la presencia pública, y una escasa organización obrera que no lograba atraer a los trabajadores: Por el contrario, el poder del Marqués de Villapesadilla, controlando, desde el Ayuntamiento y sus negocios, los principales servicios de la ciudad, aparecía como indestructible ${ }^{5}$.

Se daban en la ciudad las condiciones necesarias para que a pesar de la efervescencia social que levantó en el país la caída de Primo de Rivera y las divisiones entre los caciques locales, permaneciera pujante la estructura caciquil. No en vano empezaban a dejarse sentir las consecuencias de la crisis internacional que en los años posteriores le llevó a una aguda postración.

Signo de su decadencia fue el estancamiento de su población. Tras un continuado crecimiento durante los años diez del siglo, en 1930 la población es prácticamente idéntica a la de $1920^{6}$. Hecho que se vio acompañado, además, por una paulatina pérdida del porcentaje de población residente en la capital respecto al conjunto de la provincia. Fenómeno de tendencia inversa a la que se dio en el conjunto de la nación, donde se registró un significativo aumento de la población en las capitales de pro-

(5) Para la situación del movimiento obrero ver PANI-FICADOR, *Ni organización, ni trabajo. y •Resumen y conclusión., Acciön, 7 y 21 de junio de 1930.

La masonería gaditana estaba formada por dos logias pertenecientes cada una a una de las dos grandes obediencias presentes en España: el Grande Oriente Español y la Gran Logia Española. A la primera pertenecía la logia Hijos de Hiram, mientras que a la segurida lo estaba la Fermín Saltochea. Ambas acabarán por fusionarse, una vez proclamada la República, creando la logia Hermano Vigor, adherida a la Gran Logia Regional del Mediodía, perteneciente al Grande Oriente Español. La documentación sobre la masonería gaditana se encuentra en el Archivo Histórico nacional, sección Guerra Civil, de Salamanca.

Para la debilidad del republicanismo local se puede consultar Diego Caro Cancela, La Segunda Repriblica en Cádiz. Elecciones y partidos politicos, Cádiz, 1987, pág.58 y'Javier Tusell, La crisis del caciquismo andaluz (1923-1931), Madrid, 1977, págs. 405 y ss.

(6) En 1900, Cádiz tenía 69.382 habitantes que en 1910 se han reducido a 67.174. Sin embargo en 1920 la población ha remontado hasta 76.718. Cercana a esta cifra es la que tiene la ciudad en $1930 ; 75.769$. Cifras que son engañosas si tenemos en cuenta que en realidad se había producido un descenso de casi tres mil habitantes en el período comprendido entre 1928 y 1930 [ 78.731 en el primer año y 75.769 en el segundo]. En Paulino García Fernández, Población de los actuales términos mun icipales (1900-1981), Madrid, 1985. 
vincia, como recogen las estadísticas ${ }^{7}$. Tusell ha relacionado este estancamiento demográfico con las actitudes políticas de Cádiz, provincia en la que la comarca del Campo de Gibraltar era la zona que registraba un mayor desarrollo durante estos años y en la que, correlativamente, se encontraban los núcleos más importantes del republicanismo gaditano ${ }^{8}$.

Además, el aumento del paro, derivado de la paulatina crisis que empieza a registrarse en los más importantes sectores productivos de la ciudad, como la construcción naval y el tráfico marítimo, y la apatía organizativa ya comentada, asentaban las bases desmovilizadoras de la pequeña burguesía y del proletariado, clases en las que se apoyará el régimen republicano, que explican los resultados electorales del 12 de abril en la capital de la provincia.

\section{LA PERMANENCIA DEL CACIQUISMO}

La proclamación de la Segunda República, de forma pacífica y sin que supusiera mengua de los poderes sociales y económicos de los caciques, no supuso, de ninguna de las maneras, la desaparición de las pautas de comportamiento del antiguo régimen. El primer ejemplo de la dependencia te la población de la autoridad, antes del caciuue ahora de las nuevas autoridades, lo tenemos en la repetición de las elecciones murisipales que el 31 de mayo se celebraron en 25 localidades de la provincia, entre ellas la capital.

Los resultados arrojaron el copo de los republicanos, en 18 de estas ciudades, con un total de 349 concejales, el $82 \%$ de los posibles ${ }^{9}$. Llama la

(7) En la publicación de la Dirección General de Estadística del Censo de 1940 aparece el siguiente cuadro:

\begin{tabular}{ccccc} 
& \multicolumn{2}{c}{ P R O V. C A D I Z } & \multicolumn{2}{c}{ E S P A Nं A } \\
1900 & Pob. Hecho & Pob. Derch & Pob. Hecho & Pob. Derch. \\
1910 & 15,79 & 15,83 & 16,84 & 16,62 \\
1920 & 15,06 & 15,06 & 17,44 & 16,76 \\
1930 & 14,97 & 14,81 & 19,13 & 18,50 \\
1940 & 14,92 & 14,54 & 21,51 & 20,37 \\
& 14,62 & 14,55 & 24,41 & 23,38
\end{tabular}
En Dirección General de Estadística, Censo de la Población española de 1940, tomo 1,
Madrid, 1943.

(8) Javier Tusell, La crisis del caciquismo andaluz (1923-1931), Madrid, 1977, ’ág.295.

(9) Las localidades donde obtuvieron el copo los republicanos fueron; Alcalá de los Gazules, Algar, Algodonales, Los Barrios, Benaocaz, El Bosque, Chiclana, Chipiona, Grazalema, Medina Sidonia, Olvera, Puerto Serrano, Rota, San Fernando, Setenil, Tarifa, Vejer y Villamartín. Además en Sanlúcar de Barrameda la filiación conocida de 19 concejales es republicana y en donde los monárquicos obtuvieron representación, Cádiz y El Puerto de Santa María, esta es mínima, el $22 \%$ en la primera, su mejor resultado, y algo más del $8 \%$ en la segunda. Los datos aparecen en el cuadro IV del trabajo de Diego Caro Cancela, La Segunda Repuiblica en Cádiz. Elecciones y partidos políticos, Cádiz, 1987, pág.84. 
atención que la mayoría de estas poblaciones o bien habían elegido sus munícipes en virtud del artículo 29 de la ley electoral, aplicable cuando sólo se presentaba una candidatura, o habían votado mayoritariamente a los candidatos monárquicos en abril. En total fueron 19 las localidades que en apenas un mes, cambiaron radicalmente su voto ${ }^{10}$.

Justificar este hecho, simplemente, por la oleada de entusiasmo que había recorrido el país por el destronamiento de Alfonso XIII, no es suficiente. Haría falta un estudio pormenorizado, municipio por municipio, para saber si, como apunta Diego Caro, la mayoría de los concejales republicanos de mayo, se habían presentado en las listas monárquicas aplicando el principio de Lampedusa, de que era preciso cambiar todo para que nada cambiara ${ }^{11}$. En Cádiz capital, el vuelco fue espectacular: los monárquicos descendieron de 7451 votantes a 1931 , mientras que la conjunción republicano-socialista aumentó hasta 4275 sus 1755 votos de abril. Cifras que representó que los partidos dinásticos que habían obtenido el copo de concejalías, cuarenta, en abril las vieran reducidas a nueve.

No fue ajena a este hecho, la descomposición que se produjo en las filas monárquicas que se vieron desbordadas por unos acontecimientos externos a la dinámica provincial. Así, poco antes de la repetición de las elecciones, los seguidores de un miembro de una de las familias caciques locales, Aramburu, ingresaron en el grupo del que sería presidente de la República, Niceto Alcalá Zamora, Derecha Liberal Republicana, que se adscribió al.Partido Republicano Autónomo, que aglutinaba a los débiles grupos republicanos de la ciudad.

Del desconcierto que se produjo en las filas derechistas de la ciudad da idea que para las elecciones a Cortes Constituyentes de junio de 1931, ningún representante del cacicato gaditano estuvo presente en la lista que se opuso a la que presentaban republicanos y socialistas, en la que figuraban nuevos republicanos, y a la que se le acusó de estar elaborada siguiendo los más rancios términos caciquiles ${ }^{12}$.

(10) Estas localidades fueron; Alcalá de los Gazules, Algar, Los Barrios, Benaocaz, Cádiz, Chiclana, Chipiona, Grazalema, Medina Sidonia, Olvera, El Puerto de Santa María, Puerto Serrano, Rota, San Fernando Sanlúcar de Barrameda, Setenil,Tarifa, Villamartín y Vejer.

(11) Diego Caro Cancela, La Segunda República en Cádiz. Elecciones y partidos politicos, Cádiz, 1987, pág.83.

(12) En una nota aparecida en la prensa local la Candidatura Gaditana de Defensa de la Répública, situada a la izquierda de la Conjunción, denunciaba que la lista de ésta última había sido elaborada en el Gobierno Civil y encasillada bajos los auspicios de Sevilla, en clara referencia a la militancia radical, ligada a su líder sevillano Diego Martínez Barrio, de los dos primeros gobernadores republicanos de la provincia, José González y Fernández de la Bandera, futuro alcalde a la capital hispalense, y Gabriel González Taltabull, que tras una breve separación volvería a coincidir con su jefe cuando éste se escindió del grupo de Lerroux para formar, con un sector de los 
Pero si la representación política del antiguo régimen entró en una aparente desaparición, no lo hizo su poder económico. Además, las denuncias recogidas ponían de manifiesto que el nuevo régimen no iba a estar a salvo de la utilización de métodos de presión y coacción para acabar de implantarse. Estas dos circunstancias estuvieron presentes durante los años de República, en la vida gaditana, sobre todo cuando ésta tuvo que enfrentarse a un tercer oponente que distorsionaba sus pretensiones de establecer un régimen popular en el que junto a las clase medias, que habían dejado pasar la hora de su propia revolución, debía tener su papel el proletariado, a esta altura del siglo XX ya organizado, para cerrar así la posibilidad de una revolución social ${ }^{13}$. El tercer elemento activo, durante estos años, fue el anarcosindicalismo que tenía una fuerte implantación en la provincia gaditana, incluyendo la capital.

\section{UNA SOCIEDAD A TRES BANDAS}

Instaurada la República, la sociedad gaditana presentaba una triple división. En primer lugar, la gran burguesía mercantil, estrechamente relacionada con los terratenientes de la provincia, que a pesar de haber perdido el poder político mantenía el económico; en segundo lugar, la nueva clase emergente formada por profesionales liberales y pequeños comerciantes que, dueña, momentáneamente, de los órganos de administración recibía el apoyo, o al menos la neutralidad, de los industriales de la ciudad que esperaban, que el nuevo régimen les sacara de la crisis económica en la que se encontraban ${ }^{14}$; y, en tercer lugar, la clase obrera, prácticamente desorganizada hasta ese momento pero que iniciaba un tardío, aunque rápido, proceso asociativo por el que los sindicatos

republicanos-socialistas, Unión Republicana. En Diario de Cádiz, (EM) 27 de junio de 1931 según cita Diego Caro Cancela, La Segunda Repüblica en Cádiz.Elecciones y partidos políticos, Cádiz, 1987, pág.101. En el mismo autor se hace una referencia a este tema en la página 98 y nota 39 del capítulo III de su trabajo citado. Además, Octavio Ruiz Manjón, -Autoridades locales y partidos políticos en Andalucía durante la Segunda República., en Actas del I Congreso de Historia de Andalucia, Córdoba, 1979, tomo II, siglos XIX-XX, págs. 423-436.

(13) Para esta visión de los objetivos de la Segunda República, Santos Juliá, Manuel Azaña, una biografía política, Madrid, 1991 págs. 67-77.

(14) En este segundo sector se podría incluir al propietario de los Astilleros de la ciudad; Horacio Echevarrieta. De elevada posición económica, sus relaciones con el líder del centrismo socialista Indalecio Prieto, y Manuel Azaña le colocaba en una posición particular, bastante alejado de la cavernicola alta burguesía local. Además, la grave crisis por la que atravesaron los astilleros y su difícil situación económica durante estos años, complicada a raíz de su implicación en los sucesos de octubre de 1934, le mantuvo muy alejado de la ciudad, en una posición que se podría definir como absentista. 
cenetistas acabarían controlando los sectores económicos más importantes para la ciudad. Cada uno de ellos tenían diferentes intereses.

\section{EL CACIQUISMO TRADICIONAL}

Para los primeros, su principal preocupación fue defender sus intereses monetarios que creían amenazados tanto por la crisis, como por las medidas que pudieran tomar las nuevas autoridades. Además, quisieron recuperar el poder político que se les hacía tanto más insufrible, por cuanto representaba unos planteamientos sociales y culturales, incluyendo los religiosos, radicalmente distintos a los suyos que se pueden incluir entre los más anclados en las pautas del antiguo régimen.

Entre sus miembros destacaban quienes habían controlado la vida política y económica de la ciudad en los últimos decenios; los Carranza o los Martínez de Pinillos. Ligados a las actividades marítimas -navieras, consignatarias, flota pesquera- defendían sus intereses patronales a través de la más poderosa entidad económica de la ciudad; la Asociación Patronal del Comercio, la Industria y la Navegación (APCIN), la Patronal, en donde, a pesar de su denominación, eran los sectores marítimos quienes dominaban. En su junta directiva encontramos no sólo a estos propietarios, como el que fue su presidente en 1936, el armador Manuel Freire Costas, quien asimismo lo era de la asociación del gremio y de la Casa de Galicia, sino también a los delegados de las grandes compañías, como la Trasatlántica o la Transmediterránea, quienes, en algún caso, llegaron a ocupar importantes cargos en las entidades oficiales gaditanas ${ }^{15}$.

Era a través de estas sociedades -Cámara de Comercio, Junta de Obras del Puerto, Consorcio de la Zona Franca, Servicios Municipalizadoscómo se extendía la red de control económico de la alta burguesía ga ditana. No resulta extraño encontrar a destacados nombres en importantes cargos como el hijo de Carranza, José León, o Gabriel Matute, máximo dirigente del tradicionalismo local, en la Junta de Obras del Puerto.

Se trataba de un grupo ligado a intereses más amplios que los estrictamente locales tlos Carranza, por ejemplo, pertenecían al Consorcio Almadrabero o tenían fábricas de conservas en Italia- y que mantuvo su influencia durante estos años, pidiendo mano dura contra los alborotadores, dejando a las nuevas autoridades hacer el trabajo sucio, mientras boicoteaban tanto su legislación como que se introdujeran en

(15) Por ejemplo, Mariano P. del Pobil, delegado de la Transmediterránea, ocupó la vicepresidencia de la Cámara Oficial de Industria, Comercio y Navegación, o Juan García de Sola, delegado de la Trasatántica que llegó a la presidencia. Tanto una comó otra naviera, por medio de su delegado en la ciudad, tenían asiganadas dos vocalías en la junta directiva de la APCIN. 
los organismos económicos. Ejerciendo, paralelamente, una labor social a caballo entre el paternalismo y la caridad cristiana, e implicándose en las sucesivas tentativas golpistas contra la República ${ }^{16}$.

Pronto vieron que sus intereses económicos no iban a ser afectados más allá de una racionalización económica o de limar las injusticias más clamorosas. Así, por ejemplo, salvaron los que tenían en el Consorcio Almadrabero, que se decía iba a ser disuelto. Además, poco tardó en reconstruir una entidad política que sustituyera a las que habían desaparecido en abril. A fines de 1931, las diversas familias caciquiles gaditanas se encuentran en Acción Ciudadana, agrupación de carácter electoral, que pervivirá durante todos estos años, incluso después de que se crearan los diversos partidos derechistas en la ciudad ${ }^{17}$. En la candidatura que venció en las elecciones de noviembre de 1933 encontramos a los más destacados miembros de este grupo social; Ramón de Carranza, Martínez de Pinillos y José María Pemán, que intentaba, ahora, presentarse como un bombre de cultura, tras iniciarse en la política, al amparo de los Carranza y sus relaciones familiares con los terratenientes jerezanos, durante la Dictadura.

Resulta difícil hablar de auténticos partidos políticos en el caso de las secciones gaditanas, dominadas por la omnipresencia de los grandes propietarios locales y sus comportamientos caciquiles. Por ello no es de extrañar el celo con el que se dedicaron, una vez recuperada la representación institucional de la provincia en 1933, al amparo de la nueva

(16) La numeración de estos hechos bastarian para un trabajo propio. Ahora baste poner algunos ejemplos. Así, el nuevo delegado del Estado, que sustituía al anterior comisario regio, en el Consorcio de la Zona Franca; José Corripio del Rey, quien se había presentado, en la lista de la conjunción, a las elecciones de abril, encontró dificultades para acometer la reforma de este organismo en el que los intereses privados se mezclaban con las peticiones de subvención estatal. Parecida situación se dio en la Lonja Pesquera, cedida, para su explotación, al Ayuntamiento por la Junta de Obras del Puerto, en tiempos de la alcaldía de Carranza.

Para la política de mano dura baste recordar el uso indiscriminado de despidos e incumplimiento de leyes sociales y contratos de trabajo que se produjo durante estos años, tras algún conflicto general y, fundamentalmente, durante el segundo bienio una vez recuperado el poder político por las derechas.

La simple lectura de la prensa local de estos años nos ofrece variados y abundantes ejemplos de esa acción caritativa y/o paternalista. En ellos podemos descubrir la gran preocupación social de los Carranza por las condiciones y puestos de trabajo de los obreros gaditanos, o el alimento corporal que acompañaba al cumplimiento de ciertos deberes religiosos.

Finalmente respecto a sus actividades golpistas, cabe recordar la importante participación que tuvieron los patronos gaditanos en la sublevación de Sanjurio en agosto de 1932, a consecuencia de la cual fueron detenidos, entre otros, José León de Carranza y el presidente de la Unión patronal, carlos Derqui.

(17) Para la evolución política de las derechas gaditanas se puede consultar, Diego Caro Cancela, La Segunda Repúbilica en Cádiz. Elecciones y partidos políticos, Cádiz, 1987. 
situación política del país, a sustituir los ayuntamientos elegidos en 1931 por comisiones gestoras controladas, fundamentalmente, por el Partido Republicano Radical, decididamente escorado hacia la derecha ${ }^{18}$.

Así pues, en 1936 todavía subsistía el poder político y económico anterior a la República. Tanto es así que la gestación de la candidatura gaditana para las elecciones de febrero, se efectuó siguiendo los más rancios moldes caciquiles. En primer lugar se produjo un baile de gobernadores civiles, tres, en los dos meses anteriores a los comicios. Tenían el encargo del presidente del Consejo de Ministros que simultaneaba, significativamente, la cartera de Gobernación, de colocar el mayor número posible de candidatos centristas, fieles al presidente de la República. Tras dos intentos fallidos, tuvo que ser quien había ocupado durante la legislatúra radical-cedista el Gobierno Civil, Luis Armiñán Odriozola -que había actuado, durante su mandato, a completa satisfacción de la reacción gaditana hasta el punto de nombrarle hijo adoptivo de la provinciala persona que negociara con los notables locales su composición. Armiñán cumplió el encargo. Tras una reunión con las cabezas de los grupos, antes de partir hacia Madrid para comunicar a Portela el resultado de las negociaciones, el encasillado, pues de tal se puede hablar, proporcionó un puesto al candidato cunero, portelista, José Antonio Canals. De esta forma el Frente Antimarxista gaditano comprendía desde los monárquicos de Carranza y los tradicionalistas de Palomino Núñez, pasando por la CEDA, tanto de Cádiz como de Jerez, hasta los restos del radicalismo y el centrista gubernamental ${ }^{19}$.

La derrota, auspiciada por la participación de los anarcosindicalistas, supuso el definitivo abandono de la derecha local de la vía parlamentaria y su implicación en la militarada que se avecinaba. Porque es poco creíble que quienes estuvieron en agosto de 1932 junto a Sanjurjo, permanecieran ahora, cuando eran vox populi los preparativos golpistas, ajenos a ellos. El paréntésis que, en cierta forma, había supuesto para la estructura social de la ciudad la República - haciéndola más compleja y desvinculando a las poblaciones del control caciquil con la pe:meabilización de las decisiones administrativas y políticas ${ }^{20}$ se cerró

(18) Antes de las elecciones de febrero de 1936. en 41 municipios de la província los ayuntamientos estaban regidos por Comisiones Gestoras. De ellas 30 estaban en manos de los radicales, 10 de la CEDA y 1 por independientes.

(19) Para el intento de formar un partido centrista a partir del gobierno en Joaquín Chapaprieta, La Paz fue posible, Barcelona, 1971 y Paul Preston, La destrucción de la democracia en España, Madrid, 1981. Las diversas gestiones para la candidatura gaditana en Diego Caro Cancela, La Segunda República en Cádiz. Elecciones y partidos políticos, Cádiz, 1987.

(20) En Alejandro R.Diez Torre, „Guadalajara; la primera crisis del caciquismo., Wad-AlHayara, n.10, Guadalajara, 1983. 
simbólicamente en julio de 1936. A fines de ese mes volvía a la alcaldía Ramón de Carranza, reiniciando de esta forma un largo período en el que el cacicato se hizo hereditario, acabando por pasar la alcaldía a su hijo José León quien reinaría hasta su muerte poco antes de la transición española y la nueva restauración borbónica.

\section{LAS CLASES MEDIAS EMERGENTES}

La paradoja en la que se debatieron los miembros de las organizaciones que componían la conjunción republicano-socialista, cuando se proclamó el nuevo régimen, fue que se encontraron dueños del poder político pero huérfanos del económico. Sus más destacados representantes en 1931, antes del ingreso en sus filas de los seguidores del viejo partido Liberal, con Aramburu e Inda a la cabeza, eran profesionales liberales como el futuro alcalde Manuel de la Pinta, médico, Santiago Rodríguez Piñero, abogado, Manuel Muñoz Martínez, militar, o los profesores Rafael Argüelles y Francisco Díaz Corda.

De ellos surgieron los diversos partidos republicanos que actuaron en la ciudad. Unidos todos ellos en torno a uno, el Republicano Autónomo, pronto, la diversidad de planteamientos que aparecieron en las clases medias les separó. El Partido Radical, el de mayor tradición e implantación, se escoró hacia la derecha, al tiempo que aumentaban las dificultades para el nuevo régimen que tenía que enfrentarse, tanto a la crisis económica como a los diferentes problemas de orden público que surgieron.

En dos cuestiones se advierte el paulatino distanciamiento de gran parte de la pequeña burguesía del nuevo régimen. Por un lado la cada vez mayor oposición, con su consiguiente acercamiento a los caciques, a la República de la Unión Patronal Gaditana (UPG), entidad que agrupaba a la mayoría de los comerciantes e industriales de la ciudad; hostelería, alimentación, pequeños constructores, almacenistas y comercio en general. Sus dirigentes, como Carlos Derqui, Eladio Campe o el radical Manuel Escandón acabaron por identificarse con las posiciones de extrema derecha. Así persofificaron la falta de imaginación e iniciativa de la burguesía gaditana que se encontró perpleja ante una situación que no açababa de entender.

De otra, dominada la vida administrativa por el radicalismo, hasta su práctica desaparición en 1936, este grupo practicó métodos caciquiles, adquiridos a través de su larga singladura, que tuvieron su máxima expresión en la sustitución de los ayuntamientos por Comisiones. Gestoras de las que ya he hablado.

Prueba de esa perplejidad es el artículo que el azañista Ignacio Chilía Giráldez, propietario del periódico El Noticiero Gaditano, publicó 
en octubre de $1931^{21}$ refiriéndose a la problemática pesquera, sector que conocía como abogado. Creía que, de un lado la actuación de la alta burguesía de la ciudad preocupada por sus intereses, ponía como ejemplo el tema de la Lonja de pescado, y, de otro, la conflictividad obrera, estaba arruinando el puerto de la ciudad que se iba quedando sin barcos que buscaban otros puertos y lonjas más baratas y menos conflictivos. Reconocía la falta de vitalidad de las clases medias locales a los que pedía que salieran de su hedonismo y miraran al mar para algo más que para el placer. Finalizaba demandando que se buscara a la persona que aglutinara a las entidades vitales de la ciudad y creara el organismo que encauzara los intereses marítimos de la ciudad.

De referencias a actividades, que pueden ser calificadas como clientelistas, están llenas las cajas de documentación pertenecientes a los líderes radicales, como Lerroux o Martínez Barrio, que se custodian en el Archivo Histórico Nacional, sección Guerra Civil: peticiones de recomendaciones, informes, cartas, negocios, 'etc... Algunas de ellas tan patéticas como la carta que recibió Lerroux, desde La Línea, en la que un empleado le pedía su traslado a Ceuta, recordándole que toda su familia [29 miembros] le vota por los favores recibidos ${ }^{22}$.

\section{LA CLASE OBRERA}

Las organizaciones proletarias españolas, en los años treinta, eran dos grandes sindicatos; la UGT, socialista, y la CNT, anarcosindicalista. :Esta última, aunque apoyó la implantación del régimen republiçano buscaba una transformación social más radical que la que éste le ofrecía. Distinto es el caso del otro grupo, el socialista, que pretendía asegurar al régimen republicano. Desde su posición de grupo representativo de la clase obrera, que aplazaba indefinidamente su objetivo transformador, pretendió que aquél no perdiera su carácter reformista. Para ello era fundamental reducir, o anular, la influencia que tenía el anarcosindicalismo.

Fue lo que se intentó por medio de la legislación laboral elaborada desde el Ministerio de Trabajo que ocupaba Largo Caballero que favoreció las tácticas tradicionales de las sociedades ugetistas. Las leyes de Jura dos Mixtos, Asociaciones Profesionales, creación de Bolsas de Trabajo municipales y de las delegaciones de Trabajo, son ejemplos de estas medidas cuya puesta en práctica supuso violentos enfrentamientos con

(21) Ignacio Chilía, •El daño que nos estamos haciendo., Noticiero Gaditano, 2 de octubre de 1931.

(22) Archivo Histórico nacional, sección Guerra Civil, PS Madrid, caja 47. Otros documentos de semejantes características, referidos a Cádiz, en cajas, de misma sección, números 396 y 2045 y PS Alicante PS 88. 
los cenetistas y no fueron ajenas a su rápida radicalización. Si legítimo era el intento de los socialistas de realizar la política que creían más conveniente, no lo fue tanto su aplicación.

Comprobaron las nuevas autoridades que la Confederación tras su reorganización, de la que fue muestra la celebración del Congreso de junio de 1931 , independientemente de la línea que controlara sus organismos directivos, no sólo no se daba por satisfecha con la nueva situación política sino que también capitalizaba el descontento y la desilusión que surgía entre los trabajadores ante la lentitud de las reformas, la persistencia de su escaso nivel de vida y la dura política de orden público.

En Cádiz, ante là mayoritaria adscripción de los trabajadores a los sindicatos cenetistas, pronto empezaron a aparecer trabas para su funcionamiento. Los procedimientos fueron variados; desde atribuir intencionalidad revolucionaria a conflictos de carácter sindical o económico hasta retrasar de forma indefinida la legalización de los nuevos sindicatos que se adherían a la CNT ${ }^{23}$.

Para ello se conjugó la presión gubernamental, desde el Gobierno Civil, la de la opinión pública, identificando anarquismo con caos, y la de las organizaciones socialistas que hicieron continuos llamamientos a los trabajadores para que abandonaran la CNT ${ }^{24}$.

Aunque en la capital no se produjeron, o al menos no tengo constancia, en los pueblos de la provincia se recogen además, actuaciones desde los ayuntamientos tendentes a obligar a los trabajadores a ingresar en sociedades ugetistas, sobre todo en la comarca de la sierra, donde éstas tenían su mayor presencia ${ }^{25}$.

(23) Por ejemplo, ya en julio, el Sindicato de Construcción cenetista tuvo que hacer pública una nota en la que aclaraba que la huelga que llevaban a cabo los obreros del hormigón era por unas nuevas bases de trabajo y no tenía una finalidad revolucionaria. En El Noticiero Gaditano, 2 de julio de 1931.

El ejemplo más claro tuvo lugar durante la primera huelga general convocada en la ciudad, por una entidad que pretendía el abaratamiento de las subsistencias y los alquileres, que contó con el apoyo de la CNT gaditana, que fue presentada por las autoridades como un movimiento revolucionario anarcosindicalista, a pesar de que fue apoyada por algunas sociedades ugetistas, y le sirvió de pretexto para iniciar las numerosas y prolongadas clausuras que jalonaron las actividades de los sindicatos gaditanos. En El Noticiero Gaditano, 6 de octubre de 1931 y La Tierra 5 y 19 de octubre de 1931.

(24) Para estas actividades reiteradámente denunciadas por la CNT gaditana se pueden consultar La Tierra, 31 de octubre y 15 de noviembre de 1931.

(25) Denuncias de este tipo se dan en Algar [El Noticiero Gaditano, 8 de agosto de 1931], El Gastor [CNT, 25 de enero de 1933] y Sanlúcar de Barrameda [CNT, 4 de enero de 1933]. 


\section{LA RUPTURA DEL CACIQUISMO}

De esta forma, al continuar las prácticas caciquiles y existir un amplio movimiento anarcosindicalista, puede comprenderse mejor que se registraran en Cádiz altos porcentajes de abstención política, no solo electoral, por parte de los trabajadores. Así, el PSOE que contaba en 1931 con un total de 2162 miembros, un año más tarde sólo tiene 977 convirtiéndose en la provincia andaluza con menor número de afiliados ${ }^{26}$.

Por el contrario los sindicatos anarcosindicalistas, a pesar de las sucesivas represiones y clausuras de sus centros aumentaron sus miembros de tal forma que, en la capital por ejemplo, de 801 en 1931 pasaron a 8.453 en $1936^{27}$. La correlación entre caciquismo y desmovilización electoral, ya no social, parece evidente favoreciendo los llamamientos abstencionistas.

- La derrota de las derechas en febrero de 1936 supuso la quiebra momentánea tanto del viejo como del nuevo caciquismo. Atrás quedaban casi cinco años en los que sus prácticas habían continuado, en algún caso bajo nuevos ropajes. Ahora la situación era radical ${ }^{28}$.

Hasta junio, fueron las organizaciones obreras, que habían firmado la unidad de acción, quienes llevaron la iniciativa. Las nuevas autoridades se vieron desbordadas. La ocupación de los astilleros y la huelga general

(26) Los datos aparecen en Antonio M. Calero, Movimientos sociales en Andalucia (1820. 1936), Madrid, 1976, pág. 153.

Según esas cifras el número de agrupaciones en la provincia era de 18 en 1931 y 22 en 1932. En 1933, al primer Congreso de la Federación de Agrupaciones Socialistas de la Provincia de Cádiz, sólo acuden 11 agrupaciones. En El Pueblo, 12 de abril de 1933. Los mayores aumentos en el número de afiliados lo registraron, significativamente, las sociedades agrícolas: 8 con 2669 federados a principios de 1932 y 15 y 5.169, respectivamente, cuatro meses después. En Diego Caro Cancela, La Segunda República en Cä́diz. Eleccičzes y partidos políticos, Cádiz, 1987, págs.145 y 146.

(27) La afiliación a la CNT gaditana durante los años treinta fue la siguiente;

$\begin{array}{cccc}\text { Año } & \text { Cádiz } & \text { Provincia } & \text { Andalucía } \\ 1931 & 801 & 11997 & 104508 \\ 1932 & & & 296244 \\ 1933 & & 34842 & 252149 \\ 1934 & 7500 & 38370 & 207820 \\ 1936 & 8453 & 45323 & 149628\end{array}$

(28) Uso este término porque, al menos en el caso de Cádiz, es dudoso que existiera una situación revolucionaria. Como decían los trabajadores gaditanos, por boca de UGT y CNT, no son horas de promesas. Queremos realidades. Realidades que a la vista de sus reivindicaciones no eran revolucionarias, por lo menos de forma inmediata. En .A todos los trabajadores., Diario de Cádiz, (ET), 8 y 9 de marzo de 1936. 
convocada en su apoyo, finalizaron con la incautación, aunque fuera provisional, de la factoría de Echevarrieta ${ }^{29}$.

Durante la primavera de 1936 se produjo en la ciudad la quiebra de los poderes caciquiles que habían controlado su vida durante los años anteriores. De un lado, los vencedores de las elecciones, no fueron capaces, como ya he dicho, de imponer su política en la ciudad y estuvieron a merced de las reivindicaciones obreras. De otro, las clases patronales apostaron decididamente por la militarada que se preparaba.

Sería finalmente éstas últimas quienes triunfarían. En julio de 1936, ocupada la ciudad a sangre y fuego, se implantó un régimen que, a pesar de las transformaciones que sufrió, tanto él como la sociedad española, mantuvo hasta su desaparición, y se advierten todavía hoy, las pautas que caracterizan la estructura de poder caciquil; existencia de amigos políticos; clientelismo, preferencia, en la vida política, del pacto antes que la lucha; desmovilización electoral; desideologización; etc...

(29) En Diario de Cádiz (EM) 25 de abril y (ET) 27, 28 y 30 de abril y 4, 5, 8, 9 y 10 de mayo de 1936.

En junio, el gobierno republicano había sufrido una profunda transformación con la elevación a la presidencia de la República de Manuel Azaña y el nombramiento de Casares Quiroga como presidente del consejo de ministros. Este empezó a aplicar una política de mano dura para abordar los conflictos sociales. En Cádiz, el gobernador civil clausuró los centros obreros y detuvo a sus directivas a principios de junio con motivo de las huelgas de trabajadores de Matagorda, descargadores de pescado y la general convocada en solidaridad con las almadrabas. En Diario de Cádiz, (EM) 11, 16, 24 y 26 de junio de 1936 y Solidaridad Obrera, 20, 21 y 25 de junio de 1936. 